\title{
EXAMINING THE RELATIONS AMONG WORKING MEMORY CAPACITY, ADHD SYMPTOLOGY AND CONSCIOUS EXPERIENCE
}

\author{
A thesis presented to the faculty of the Graduate School of \\ Western Carolina University in partial fulfillment of the \\ requirements for the degree of Master of Arts in Clinical Psychology \\ By \\ Leila Annie Beikmohamadi \\ Director: Dr. Matt Meier \\ Assistant Professor of Psychology \\ Department of Psychology \\ Committee Members: Dr. David de Jong, Psychology \\ Dr. David Solomon, Psychology
}

April 2021 


\section{TABLE OF CONTENTS}

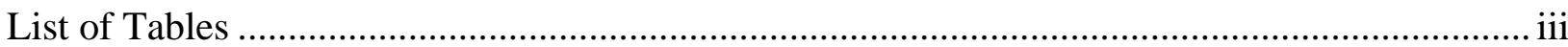

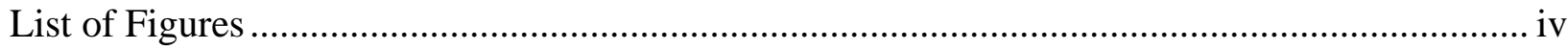

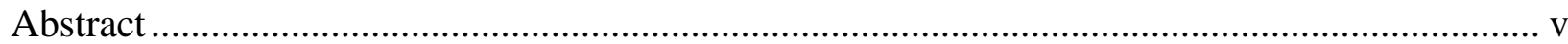

Chapter One: Literature Review .......................................................................................... 1

Working Memory Capacity as a Representation of Executive Control....................................... 4

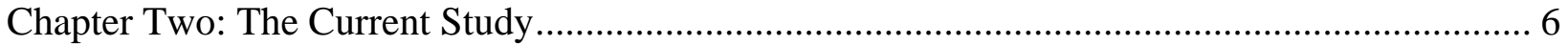

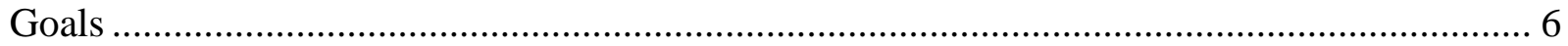

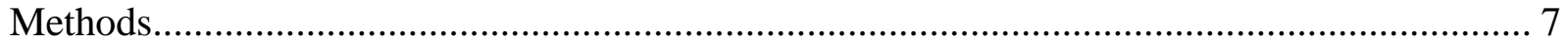

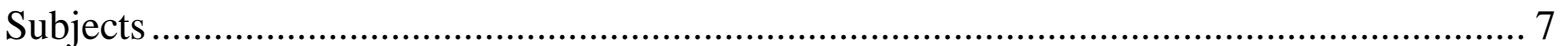

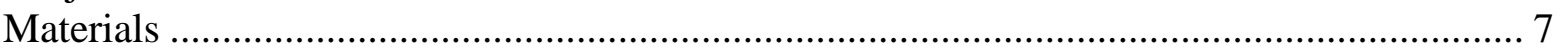

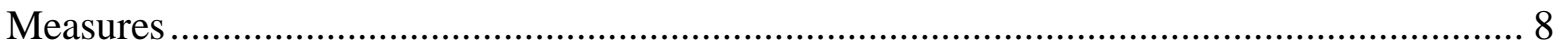

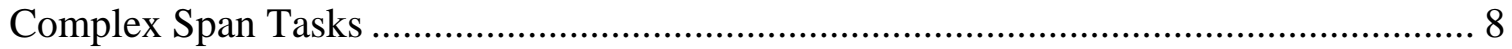

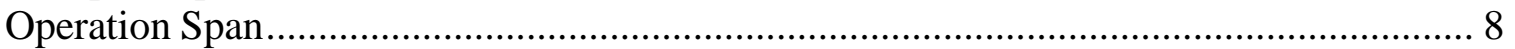

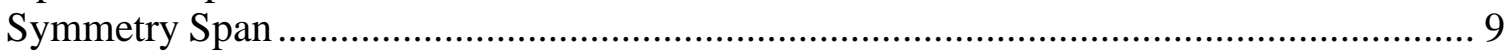

Sustained Attention to Response Task ……………………………………………... 10

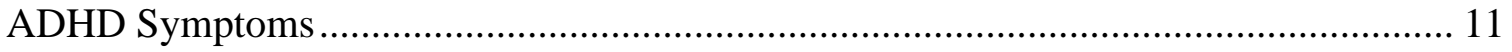

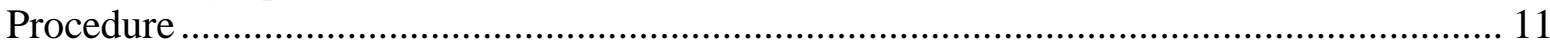

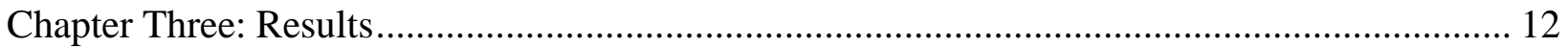

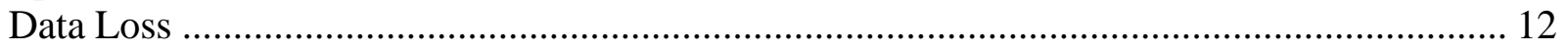

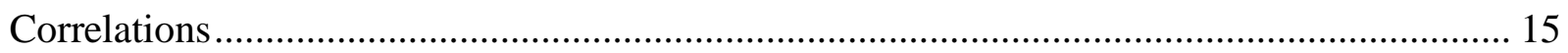

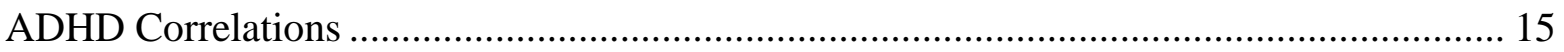

Working Memory Capacity Correlations.............................................................................. 21

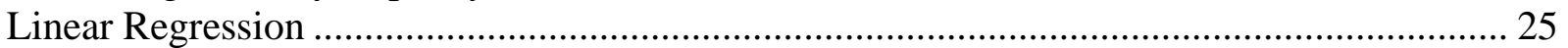

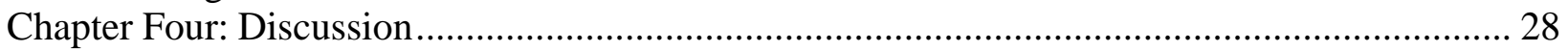

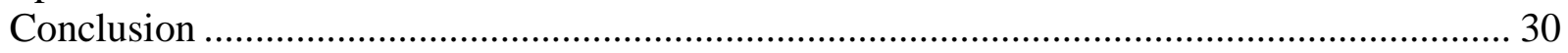

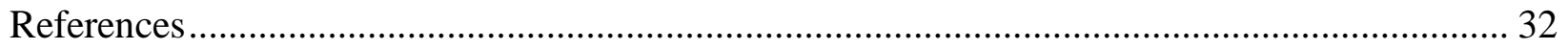

Appendix A: Operation Span Complex Span Task (OSPAN)................................................. 38

Appendix B: Symmetry Complex Span Task (SYMSPAN) …………………........................ 39

Appendix C: Sustained Attention to Response Task (SART) ......................................................... 40

Appendix D: Sustained Attention to Response (SART) Task Instructions ................................... 41

Appendix E: ADHDRS-IV Self-Report Questionnaire ............................................................. 44

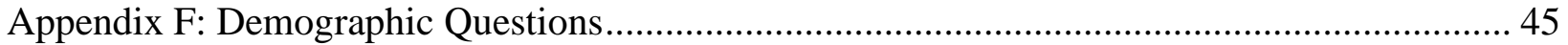




\section{LIST OF TABLES}

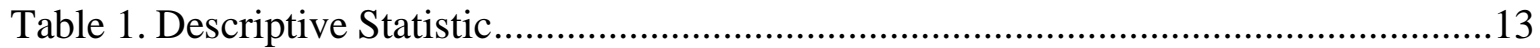

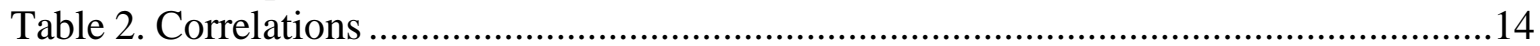

Table 3. Linear Regression of WMC and Total ADHD Symptoms on each Thought Probe

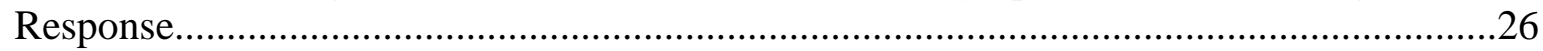




\section{LIST OF FIGURES}

Figure 1. Correlation between thought probes and total ADHD symptoms .........................18

Figure 2. Correlation between thought probes and inattention symptoms...........................19

Figure 3. Correlation between thought probes and hyperactivity symptoms.......................20

Figure 4. Correlation between thought probes and working memory capacity (WMC)......22

Figure 5. Correlations of executive dependent and with ADHD symptoms and WMC......23

Figure 6. Correlations of non-executive dependent and with ADHD symptoms and WMC

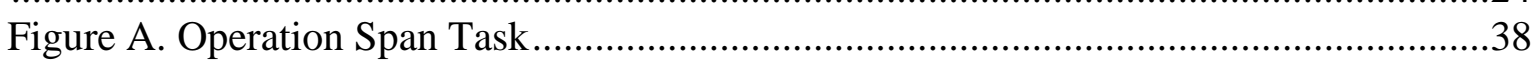

Figure B1. Presentation Phase of the Symmetry Span Task ………………………............39

Figure B2. The Recall Phase of the Symmetry Span Task ..................................................39

Figure C. Sustained Attention to Response Task ................................................................23 


\begin{abstract}
EXAMINING THE RELATIONS AMONG WORKING MEMORY CAPACITY, ADHD SYMPTOLOGY AND CONSCIOUS EXPERIENCE

Leila Beikmohamadi, M.A.

Western Carolina University (April 2021)

Director: Dr. Matt Meier
\end{abstract}

An operational definition of mind wandering is when one has thoughts unrelated to the current task(s) (Smallwood \& Schooler, 2015). Mind blanking can be thought of as a subtype of mind wandering where there is an inability to report the content of these task-unrelated thoughts. Van den Driessche et al. (2017) found that children and young adults with more AttentionDeficit/Hyperactivity Disorder (ADHD) symptoms report more mind blanking than those with fewer ADHD symptoms and that non-medicated children with ADHD reported less mind wandering and more mind blanking than medicated children with ADHD. Van den Driessche et al. speculated that medication facilitated executive control and that executive resources support mind wandering (and on-task thought). These findings also bear on the theoretical debate on the role of executive functions in conscious experience. Some argue that executive functions support mind wandering (Levinson et al., 2012; Smallwood, 2010), while others argue and that mind wandering situationally results from a lack (or failure) of executive control (McVay \& Kane, 2010; Meier, 2019). In this study, with a young adult sample, I tested the association between ADHD symptomology and conscious experience and if executive resources moderate the proportion of reporting mind blanking and mind wandering. The current study found evidence for Van den Driessche et al.'s finding of a positive and significant association between mind 
blanking and ADHD symptomology. The current study was also broadly consistent with Van den Driessche et al.'s finding of an ADHD-related trade-off involving mind blanking, but importantly differs from Van den Driessche et al. in that mind wandering was not involved in this trade-off. The current study did not find an association between working memory capacity and mind wandering. Thus, I found no evidence for executive resources supporting mind wandering, consistent with previous studies (McVay \& Kane, 2009, 2012a, 2012b; Meier, 2019; Robison \& Unsworth, 2018; Unsworth \& McMillan, 2013, 2014). 


\section{CHAPTER ONE: LITERATURE REVIEW}

Have you ever been driving home from a long day of work and catch yourself re-playing an interaction you had with a co-worker earlier that day? Your attention is devoted to remembering that interaction and not towards the task at hand (i.e., driving). Hereafter, I refer to having thoughts like these, thoughts unrelated to the current task being carried out, as mind wandering (Smallwood \& Schooler, 2015). If you were mind wandering, you may not be able to provide a report of the content of the off-task thoughts you were having. An inability to report thought content is mind blanking (Ward \& Wegner, 2013). Mind blanking is distinguished from mind wandering by the failure to report the subject (or contents) of the off-task thoughts. That is, during mind blanking, there is either no (clear) thought or a lack of conscious access to any thought. People can distinguish between mind blanking and mind wandering. Ward and Wegner (2013) demonstrated mind blanking and mind wandering are distinct by having subjects complete a reading task and probing them with questions about their mind states at random points during the task. Subjects reported both mind blanking and mind wandering, showing that the two states are distinguishable phenomenological experiences. Also, the two mind states were associated with different behavioral outcomes. In self-paced tasks, mind wandering impaired reading comprehension, whereas mind blanking did not.

Van den Driessche et al. (2017) examined conscious experience in four groups of children (ages 6-12): children diagnosed with Attention-Deficit/Hyperactivity Disorder (a neurocognitive disorder marked by symptoms of impulsivity and hyperactivity ([American Psychiatric Association, 2013]; ADHD) taking medication, children diagnosed with ADHD not taking medication, a control group with other psychiatric disorders, and a healthy control group 
(Experiment 1). Additionally, Van den Driessche et al. compared the conscious experience between a young adult population $(M$ age $=24)$ that self-reported more ADHD symptoms (a score of five or higher on the DIVA [Kooij \& Francken, 2010] ADHD self-report questionnaire) and a control population that self-reported fewer ADHD symptoms (a score of less than five on the DIVA [Kooij \& Francken, 2010] self-report questionnaire; Experiment 2). Subjects completed a sustained attention to response task that had embedded thought probes. Van den Driessche et al. examined the following thought reports in both experiments: on-task focus, taskrelated interference (lapse of attention due to thinking about something related to the task), distraction (interference caused by something in the environment), mind wandering, and mind blanking. The authors tested if thought reports differed by group membership.

In Experiment 1, Van den Driessche et al. found that the non-medicated ADHD group had increased mind blanking, but not mind wandering relative to the medicated ADHD group and the two control groups. The medicated ADHD group had more mind wandering than the other three groups (i.e., non-medicated ADHD, other psychiatric disorders, healthy controls). In addition, the non-medicated ADHD group had less mind wandering and on-task thoughts, which were combined to form a composite (both mind wandering and on-task thought are theorized to require executive resources; Smallwood, 2013) compared to the medicated ADHD group and control groups. The medicated ADHD group's mind blanking levels were at the same level as children who did not have ADHD, suggesting that the medication reduced mind blanking and increased mind wandering. No significant differences were found among the groups in the percentages of reports speculated not to require executive resources (i.e., a composite formed of task-related interference and distraction). 
In Experiment 2, the young adults who reported more ADHD symptoms had more mind blanking than the young adults who reported fewer ADHD symptoms. There was not a significant difference in mind wandering reports between the two groups. Similar to Experiment 1, the group that reported more ADHD symptoms had less mind wandering and on-task thoughts (combined into a composite) compared to the group that reported fewer ADHD symptoms. Also like Experiment 1, there were no statistically significant differences between the two groups in the percentage of reports speculated not to require (or require little) executive resources (i.e., task-related interference and distraction).

Based on their results and previous reports of ADHD associated with deficits in executive functioning (working memory, self-regulation, behavior analysis, and internalization of speech; Barkley, 1997), Van den Driessche et al. speculated that increased mind blanking in those who have more ADHD symptomology is due to a lack of (or ineffective use of) executive resources. More specifically, those who lack executive resources may also lack the ability to maintain an internal train of thought, whether applied to mind wandering or on-task thought. Van den Driessche et al. also speculated that attention to external tasks might not depend solely on executive function but also other factors, such as motivation. Motivation is weaker in ADHD (Volkow et al., 2011). In this context, motivation issues in those with ADHD refer to dysfunction in reward processing. More specifically, those with ADHD may require larger amounts of reinforcement to perform well on tasks than those without ADHD (Haenlein \& Caul, 1993; Johansen et al., 2009). Moreover, higher motivation levels reduce mind wandering separately from executive control (Mrazek et al., 2012). It seems motivation is required to sustain attention to a task rather than letting thoughts wander away from it. 
Van den Driessche et al.'s finding of elevated mind blanking in those with non-medicated ADHD is in line with work by Franklin et al. (2017), who showed that ADHD scores were positively correlated with the frequency of mind wandering and a lack of meta-awareness. In Franklin et al. (2017), meta-awareness was defined as recognizing off-task thoughts, and a lack of meta awareness was revealed by reporting that one was unaware of mind wandering when prompted in daily life by a personal digital assistant. Van den Driessche et al.'s finding of increased mind blanking in those with ADHD symptomology also aligns with work that has described ADHD-associated metacognitive deficits more broadly. Antshel and Nastasi (2008) showed that preschoolers with ADHD had metamemory skills that lagged behind the preschoolers without ADHD and Knouse et al. (2005) found that adults with ADHD exhibited impaired metacognitive monitoring by overestimating their abilities in driving scenarios compared to control groups.

Additionally, Van den Driessche et al.'s result that methylphenidate increased mind wandering, which they interpreted as being due to increased executive resources, lends support for the claim that mind wandering (and on-task focus) requires executive resources. There is an active debate on the role of executive functions in mind wandering. Some claim that mind wandering requires executive resources, implying mind wandering will be greater in situations that demand fewer resources, and people who have more resources available will mind wander more than people with less available resources (Levinson et al., 2012; Smallwood, 2010). Others argue and provide evidence that in situations where someone is trying to concentrate, mind wandering occurs due to executive functions' failure to maintain control over attention (McVay \& Kane, 2010; Meier, 2019).

\section{Working Memory Capacity as a Representative of Executive Control}


In the current study, I used working memory capacity as a measure of executive functioning. Working memory capacity is an executive function essential for sustaining, manipulating, and retrieving information to service ongoing cognition (Unsworth \& Robison, 2020). A latent variable composed of complex span tasks correlated at $r=.97$ with a latent variable of executive function tasks (e.g., mental arithmetic, mental control, verbal fluency, Wisconsin Card Sorting Test; McCabe et al., 2010). In addition, a negative association between working memory capacity and mind wandering has frequently been reported. For example, Kane et al. (2007; replicated by Kane et al., 2017) tested undergraduates' working memory with complex span tasks in the lab. Subjects also provided thought reports and information about their context in their daily lives over seven days using personal digital assistants that prompted students to report their thoughts throughout the day. Variation in working memory capacity was contextually associated with the amount of mind wandering. In situations where subjects were trying to concentrate, subjects with lower working memory capacities mind wandered more than subjects with higher working memory capacities. This finding of a negative association between working memory capacity and the propensity to mind wander has been shown several times in the lab (e.g., McVay \& Kane, 2009, 2012a, 2012b; Meier, 2019; Robison \& Unsworth, 2018; Unsworth \& McMillan, 2013, 2014). 


\section{CHAPTER TWO: THE CURRENT STUDY}

\section{Goals}

The proposed study attempted to replicate and extend the findings of Van den Driessche et al.'s (2017) experiments 1 and 2. Here, using a young adult population (like Van den Driessche et al. Experiment 2), I attempted to replicate the associations between ADHD symptoms and thought reports with a specific interest in the critical ADHD-mind blanking association. To extend Van den Driessche et al., I also estimated associations between thought reports and the hyperactivity and inattentive ADHD subscales. To help assess Van den Driessche et al.'s speculation that medication recruited executive resources that in turn affected thought reports (i.e., conscious experience), I measured executive function directly by assessing working memory capacity with two shortened complex span tasks. Following from Van den Driessche et al., I formed composites from thought reports assumed to require executive resources (i.e., mind wandering and on-task; Smallwood, 2013), and from thought reports assumed to require no (or little) executive resources (i.e., distraction and task-related interference) and tested for associations between these composites and working memory capacity and ADHD. If Van den Driessche's speculation about medication and executive function is correct, working memory capacity should positively correlate with mind wandering and the presumed executive-function composite and negatively associate with mind blanking. In addition to testing these associations, I assessed an alternate model of associations among these variables with working memory capacity and ADHD symptomology both allowed to predict unique variance in thought reports and a model term estimating the interaction between working memory capacity and ADHD 
symptoms. This model differs from the one implied by Van den Driessche et al. where ADHD was used as a proxy for executive functioning.

\section{Methods}

This study was preregistered on August $28^{\text {th }}, 2019$ (https://aspredicted.org/6s7wm.pdf). The data for this study was collected during the Fall 2019 semester.

\section{Subjects}

Three hundred and two subjects from Western Carolina University completed the informed consent for this study (mean incoming student SAT scores range from 1116 to 1149 for cohorts entering Fall 2017 through Fall 2019). Of these 302 subjects, 59\% were female. Subjects had a mean age of $19(S D=2)$; Of the subjects who gave ethnicity information (two subjects declined), 248 identified as white, 20 as black, 10 as multiracial, 10 as Asian, seven as Native American or Alaskan Native, and five as other. Subjects received partial credit for a course requirement as compensation for their participation. The stopping rule for data collection was the end of a semester in which I had at least data from 250 subjects. Data collection was completed during the 2019 fall semester. This sample size was chosen on the basis that correlations ( $\rho$ s) as weak as .10 stabilize within a narrow window when approaching 250 subjects (Schönbrodt \& Perugini, 2013), thus allowing precise estimates. The advertised eligibility criteria for participation in this study were being within the age range of 18-30, having no serious visual impairments, and being native English speakers. Subjects attended one session that lasted up to one hour.

\section{Materials}

All measures were programmed in E-Prime (Psychology Software Tools, Pittsburg, PA, 
2012) on Windows computers, and subjects used a standard mouse and QWERTY keyboard on liquid crystal display monitors.

\section{Measures}

Complex Span Tasks. Working memory capacity was tested with two shortened complex span tasks (Foster et al., 2015) to limit each experimental session's time and maximize the number of subjects. Subjects completed one block of each of the two shortened complex span tasks (compared to the standard three-block complex span task). In these tasks, subjects memorized the identity and order of stimuli (depictions of these tasks are in appendix A and B). In between each item to remember, subjects completed an unrelated task in which they gave a true/false answer within a specific timeframe. After each sequence, which had a random amount of trials (with differing set sizes for each complex span variant), subjects recalled the memory items. Before administrating the scored trials, subjects practiced both parts of the task individually and all together. In the first part, subjects practiced the recall part of each task; they memorized 2-3 of the recall items with no processing task. In the second part, subjects completed the processing task with no recall items. Also, in this section, the average response time was calculated. If a subject's response time was 2.5 standard deviations away from their mean response time during the scored trials, the computer counted that processing task as an error and moved to the next screen. A working memory composite was created by averaging the z-scores of both the symmetry and operation span tasks.

Operation Span (Unsworth et al., 2005). The operation span task first had subjects practice memorizing letters (between 2-3 letters). Then subjects practiced solving simple math problems (e.g., $[3 * 4]+2=$ ?) through answering them in a true-false style. In the scored task, subjects solved a simple math problem which afterward an answer would appear (half were true). 
Subjects then decided if that answer was true or false in a certain time frame. After the math problem, subjects were presented with a letter, out of twelve possible letters, to remember. Each sequence had a random length between 3 and 7 problem-letter pairs. One trial of each set size (i.e., 3-7) was presented, for a total of five trials. During the recall phase, subjects were presented with 12 possible letters with boxes next to each of them. Subjects were instructed to click on the boxes next to the letters they saw in a trial with a computer mouse in the order that the letters appeared. Scores on this task were computed by adding the total number of letters remembered in the correct serial order (Conway et al., 2005). There was a maximum score of 25 for this task and Cronbach's $\alpha$ for one block of this task has been reported as .69 (Foster et al., 2015). This task took approximately 15 minutes to complete (see Appendix A for diagram of the task).

Symmetry Span (Kane et al., 2004). The symmetry span task first had subjects practice memorizing the position and sequence of colored squares on a 4 x 4 grid, practice deciding if a picture was vertically symmetrical, and then practicing both together. In the scored task, subjects were presented with a picture in which they decided if it was symmetrical, and afterward, one cell filled in the $4 \times 4$ matrix would appear on the screen for $650 \mathrm{~ms}$. Each sequence had a random length between 2 and 5 trials where there was one of each trial size. During the recall phase, subjects clicked on cells in an empty matrix to show the location and sequence of the red squares they saw on the previous screens by clicking on the cells in an empty matrix. Set sizes ranged from two to five, and there was one of each set size, which was presented in random order. There was a maximum score of 14 for this task. Cronbach's $\alpha$ for one block of this task has been reported as .61 (Foster et al., 2015). This task took approximately 10 minutes to complete (see Appendix B for diagram of the task). 


\section{Sustained Attention to Response Task (SART) (Kane et al., 2016). Subjects}

completed a Sustained Attention to Response Task (SART). This was a no/no-go task in which subjects pressed the space bar every time they saw a member from the non-target category and withheld a response when they saw words from the target category. Subjects practiced one trial where they pressed the space bar for boys' names and withheld responses for girls' names. The SART task was divided into four blocks, each of which had three miniblocks of 45 trials accumulating to 540 total trials. During each miniblock, subjects were presented with five target stimuli (vegetable names) and 45 non-target stimuli (animal names). Each word was presented on-screen for $300 \mathrm{~ms}$ and then was immediately covered by a string of "X"'s for $1500 \mathrm{~ms}$. This task took approximately 20 minutes to complete (see Appendix $\mathrm{C}$ for response and withhold diagram). The dependent variable for this part of the task was $d$ ' (i.e., hit rate to animals minus false alarm rate to vegetables) and the standard deviation $(S D)$ of response times to "go" (animal) trials.

Within the SART, subjects responded to thought probes. There were 9 probes per block for a total of 36 probes. Subjects were asked to evaluate their thoughts with the prompt, "What were you just thinking about?" and press a number that best matches their thoughts. Response options included: "1: On task," "2: Task/Task performance," "3: Distraction," "4: Mind wandering," and 5: Mind blanking." Subjects were briefed on what each of these responses meant after finishing the practice trial at the beginning of the task (see Appendix D for task instructions). I summed up how many times each thought response was indicated and create proportions. I created composites of thought responses that indicate executive resources were used (mind wandering and on-task thinking) and thought responses that are not supposed to indicate executive resources (task-related interference and distraction). 
ADHD Symptoms. Subjects completed a self-report questionnaire from the ADHDRSIV (DuPaul et al., 2016). Subjects answered 18 questions about if they had ADHD symptoms within the past 6 months. Response options for all the questions were: never or rarely, sometimes, often, and very often. Nine items in the questionnaire assess the inattention subscale, and nine items assess hyperactive-impulsive symptoms (DuPaul et al., 2016). Scores were summed for these subtypes and an overall score. This task took approximately 5 minutes to complete (see Appendix E for instructions and questions).

\section{Procedure}

Subjects started the session by reading and signing an informed consent form. In accordance with Van den Driessche et al. (2017), the first two tasks of the session were the SART and the ADHD questionnaire (and these were counterbalanced as in Van den Driessche et al. [2017]). Following these first two tasks, subjects completed the operation span task and the symmetry span task (all subjects completed these tasks in the same order). Finally, subjects completed a demographics questionnaire (see Appendix F for instructions and questions). The sessions took no longer than 1 hour to complete. 


\section{CHAPTER THREE: RESULTS}

Planned analyses for this thesis are contained in the prospectus (https://osf.io/kw3hu/) and are a subset of the analyses in the preregistration (https://aspredicted.org/6s $7 \mathrm{wm} . \mathrm{pdf})$. All analyses were carried out using JASP version 0.14.1 (University of Amsterdam, Amsterdam). I used an alpha level of .05 for null hypothesis significance testing.

\section{Data Loss}

I made all data exclusions per the preregistration. I excluded eight subjects from analyses who did not meet the SART response time standard deviation inclusion criterion, and two subjects whom experimenters deemed as noncompliant with instructions across tasks. These noncompliance decisions were made without consulting the subjects' data. Because of computer or experimenter error, I am missing data from two subjects in the symmetry span, and one subject on the ADHDRS-IV. Thus, I included data from 292 subjects from the SART and operation span tasks, data from 291 subjects for the ADHDRS-IV, and 290 subjects from the symmetry span. Descriptive statistics for all dependent variables can be seen in Table 1. Intercorrelations for measures can be seen in Table 2 . 
Table 1

Descriptive Statistics

\begin{tabular}{|c|c|c|c|c|c|c|c|}
\hline Variable & Mean & $\begin{array}{l}\text { Std. } \\
\text { Deviation }\end{array}$ & Minimum & Maximum & Skew & Kurtosis & $\mathrm{N}$ \\
\hline SART d' & 1.55 & 1.12 & -.46 & 4.51 & .19 & -.83 & 292 \\
\hline On-Task Proportion & .30 & .20 & .00 & .97 & .70 & .11 & 292 \\
\hline Task-Related Proportion & .30 & .19 & .00 & .92 & .63 & -.05 & 292 \\
\hline Distraction Proportion & .10 & .10 & .00 & .86 & 2.83 & 15.61 & 292 \\
\hline Mind Wandering Proportion & .19 & .13 & .00 & .69 & .62 & .10 & 292 \\
\hline Mind Blanking Proportion & .11 & .13 & .00 & .69 & 1.87 & 4.20 & 292 \\
\hline Operation Span Task & 15.43 & 5.74 & 2.00 & 25.00 & -.18 & -.82 & 292 \\
\hline Symmetry Span Task & 8.62 & 3.03 & 1.00 & 14.00 & -.12 & -.68 & 290 \\
\hline WMC & .00 & .82 & -1.84 & 1.72 & -.09 & -.46 & 290 \\
\hline Total ADHD & 15.61 & 7.62 & 1.00 & 50.00 & .90 & 1.49 & 291 \\
\hline Inattention & 7.89 & 4.69 & .00 & 26.00 & .91 & .95 & 291 \\
\hline Hyperactivity & 7.73 & 3.96 & .00 & 24.00 & .87 & .99 & 291 \\
\hline
\end{tabular}

Note: SART = Sustained Attention to Response Task; WMC = Working Memory Capacity. 
Table 2

Correlations

\begin{tabular}{|c|c|c|c|c|c|c|c|c|c|c|c|c|c|c|}
\hline Variables & 1 & 2 & 3 & 4 & 5 & 6 & 7 & 8 & 9 & 10 & 11 & 12 & 13 & 14 \\
\hline 1. Working Memory Capacity & - & & & & & & & & & & & & & \\
\hline 2. Operation Span & .82 & - & & & & & & & & & & & & \\
\hline 3. Symmetry Span & .81 & .33 & - & & & & & & & & & & & \\
\hline 4. SART d' & .17 & .10 & .18 & - & & & & & & & & & & \\
\hline 5. On-Task & .03 & -.01 & .05 & .45 & - & & & & & & & & & \\
\hline 6. Task-Related Interference & .05 & .10 & -.03 & -.16 & -.42 & - & & & & & & & & \\
\hline 7. Distraction & -.13 & -.12 & -.09 & -.06 & -.16 & -.28 & - & & & & & & & \\
\hline 8. Mind Wandering & .02 & -.06 & .09 & -.07 & -.42 & -.31 & -.01 & - & & & & & & \\
\hline 9. Mind Blanking & -.04 & .00 & -.06 & -.33 & -.38 & -.29 & -.09 & .09 & - & & & & & \\
\hline 10. Executive Composite & .04 & -.05 & .12 & .43 & .77 & -.67 & -.17 & .26 & -.35 & - & & & & \\
\hline 11. Non-Executive Composite & -.01 & .05 & -.07 & -.20 & -.51 & .87 & .23 & -.32 & -.34 & -.77 & - & & & \\
\hline 12. Total ADHD & .03 & .01 & .04 & -.15 & -.15 & -.09 & .08 & .11 & .20 & -.08 & -.05 & - & & \\
\hline 13. Inattention ADHD & .04 & .01 & .06 & -.15 & -.18 & -.05 & .01 & .11 & .21 & -.11 & -.04 & .90 & - & \\
\hline 14. Hyperactivity ADHD & .01 & -.00 & .01 & -.11 & -.07 & -.17 & .14 & .07 & .12 & -.03 & -.05 & .86 & .55 & - \\
\hline
\end{tabular}

Note: SART = Semantic Sustained Attention to Response Task; Executive Composite = On-task and Mind Wandering combined;

Non-Executive Composite $=$ Task-Related Interference and Distraction combined; ADHD = Attention-Deficit/Hyperactivity Disorder; Variables 5-9 are proportion of thought probe response. 


\section{Correlations}

\section{ADHD Correlations}

For all correlations, in addition to examining them with a frequentist interpretation, I also examined them using Bayes Factors (BF). The BF indicates if the correlations are more likely from a point-null distribution (the null hypothesis) or if it came from a Cauchy distribution where $50 \%$ of the distribution lies between -.33 and .33 (alternative hypothesis). Here, numbers greater than one support the alternative hypothesis, and numbers less than one support the null hypothesis that the correlation is more likely from the point-null distribution $(r=0)$.

All correlations are displayed in scatterplots below (see Figure 1 for total ADHD correlations, Figure 2 for inattention symptoms correlations, and Figure 3 for hyperactivity symptoms correlations). Van den Driessche et al. did not estimate correlations with inattentive and hyperactivity subscales. Consistent with Van den Driessche et al., the proportion of mind blanking reports was significantly positively correlated with the total amount of ADHD symptoms, $r(289)=.20, p<.001,95 \%$ Confidence Interval $(C I)[.08, .30], B F_{10}=32.38$. Mind blanking significantly positively correlated with inattention symptoms, $r(289)=.21, p<.001, C I$ $[.10, .32], B F_{10}=102.24$, and hyperactivity symptoms, $r(289)=.12, p=.04, C I[.01, .23], B F_{10}$ $=1.12$. As can be seen in Figure 1 Panel A, an outlier may have exerted influence on the total ADHD estimate. When this outlier was removed, mind blanking thought reports were positively associated with total amount of ADHD symptoms, $r(288)=.14, p=.02, C I[.03, .25], B F_{10}=$ 2.17, inattention symptoms, $r(288)=.17, p=.004, C I[.06, .28], B F_{10}=7.74$, but not with hyperactivity symptoms, $r(288)=.07, p=.26, C I[-.05, .18], B F_{10}=.26$.

There was a significant difference between groups in mind wandering as a function of ADHD symptoms in Van den Driessche et al.'s Experiment 1 but not their Experiment 2. There 
were no significant differences among any of the other thought report categories as a function of ADHD status. Here, consistent with Van den Driessche et al.'s Experiment 2, the proportion of mind wandering was not associated with the total amount of ADHD symptoms, $r(289)=.11, p=$ $.07, C I[-.01, .22], B F_{10}=.68$, Mind wandering was also not associated with inattention symptoms, $r(289)=.11, p=.054, C I[-.00, .23], B F_{10}=.85$, or hyperactivity symptoms, $r(289)=$ $.07, p=.24, C I[-.05, .18], B F_{10}=.27$. Inconsistent with Van den Driessche et al.'s null results, the proportion of on-task thought reports was significantly negatively associated with the total amount of ADHD symptoms, $r(289)=-.15, p=.01, C I[-.26,-.03], B F_{10}=3.13$. On-Task thought reports were also significantly negatively associated with inattention symptoms, $r(289)=$ $-.18, p<.001, C I[-.28,-.06], B F_{10}=11.24$, but were not associated with hyperactivity symptoms, $r(289)=-.08, p=.19, C I[-.19, .04], B F_{10}=.32$. Consistent with Van den Driessche et al., task-related interference thought reports were not associated with total ADHD symptoms, $r(289)=-.09, p=.14, C I[-.20, .03], B F_{10}=.41$. Task-related interference thought reports were also not associated with inattention symptoms, $r(289)=-.05, p=.45, C I[-.16, .07], B F_{10}=.18$, but were significantly negatively associated with hyperactivity symptoms, $r(289)=-.12, p=$ $.048, C I[-.23,-.001], B F_{10}=.93$. Also consistent with Van den Driessche et al., distraction thought reports were not associated with total ADHD symptoms, $r(289)=.08, p=.19, C I[-.04$, $.19], B F_{10}=.31$. Distraction thought reports were also not associated with inattention symptoms, $r(289)=.01, p=.86, C I[-.10, .13], B F_{10}=.14$, but were positively associated with hyperactivity symptoms, $r(289)=.14, p=.02, C I[.02, .25], B F_{10}=1.83$. As can be seen in Figure 1 Panel E, Figure 2 Panel E, and Figure 3 Panel E, an outlier may have exerted influence on these estimates. When this outlier was removed, distraction thought reports were still not significantly associated with total ADHD symptoms, $r(288)=.01, p=.90, C I[-.11, .12], B F_{10}=.14$, and inattention 
symptoms, $r(288)=-.05, p=.41, C I[-.16, .07], B F_{10}=.19$. The association between distraction and hyperactivity symptoms became non-significant, $r(288)=.07, p=.21, C I[-.04, .19], B F_{10}=$ .29 .

Following Van den Driessche et al., I created two composites. One composite was composed of thought reports deemed to require executive resources (mind wandering and ontask) and the second was thought reports deemed not to require executive resources (task interference and distraction). I tested the correlations between composites and total ADHD symptomology (see Figures 5 and 6). Inconsistent with Van den Driessche et al.'s Experiments 1 and 2, the executive dependent composite was not associated with the total amount of ADHD symptoms, $r(289)=-.08, p=.16, C I[-.20, .03], B F_{10}=.36$. The executive dependent composite was also not associated with inattention symptoms, $r(289)=-.11, p=.07, C I[-.22, .01], B F_{10}=$ .69 , or hyperactivity symptoms, $r(289)=-.03, p=.58, C I[-.15, .08], B F_{10}=.16$. Consistent with Van den Driessche et al.'s Experiments 1 and 2, the non-executive resource was not associated with the total amount of ADHD symptoms, $r(289)=-.05, p=.40, C I[-.16, .06], B F_{10}=.20$. There were also no associations between the non-executive resource composite and inattention symptoms, $r(289)=-.04, p=.50, C I[-.16, .08], B F_{10}=.17$, nor hyperactivity symptoms, $r(289)$ $=-.05, p=.41, C I[-.16, .07], B F_{10}=.19$. 


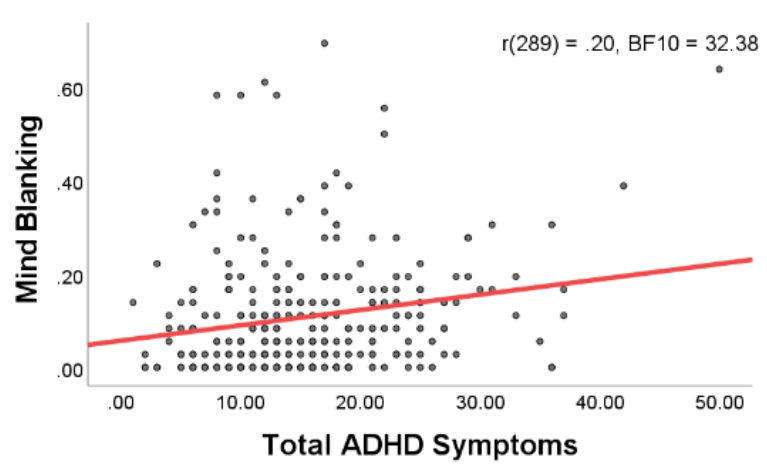

(A)

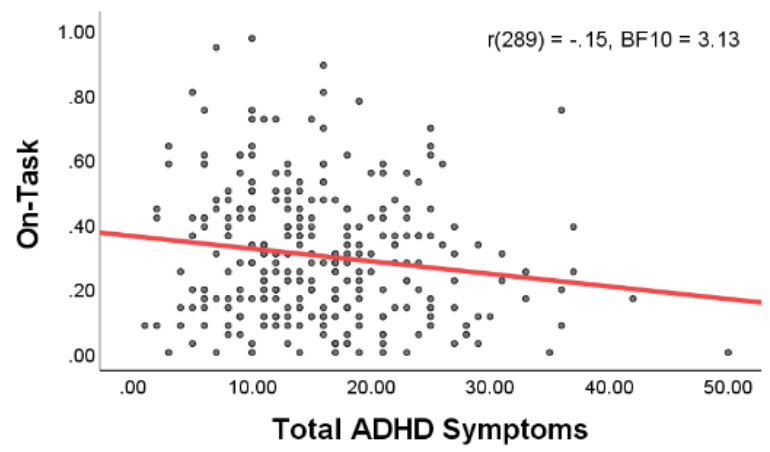

(C)

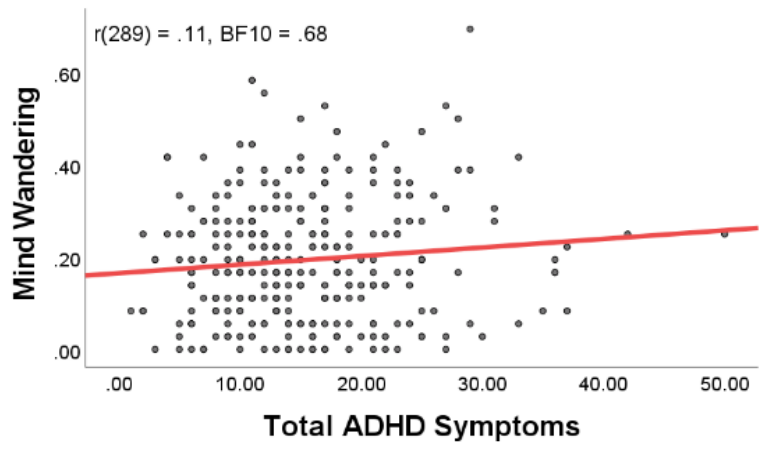

(B)

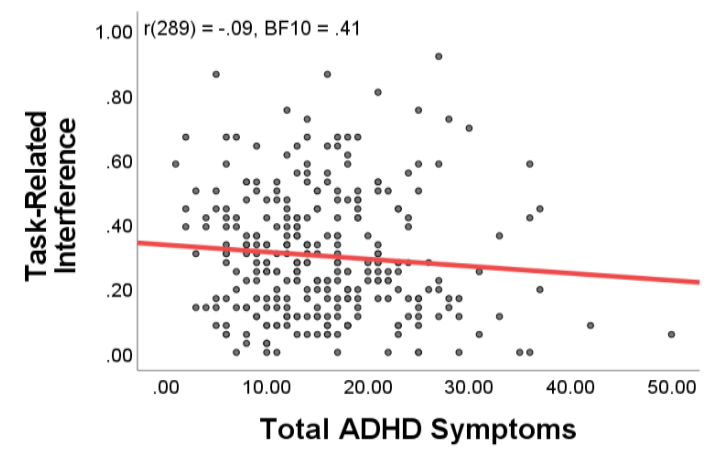

(D)

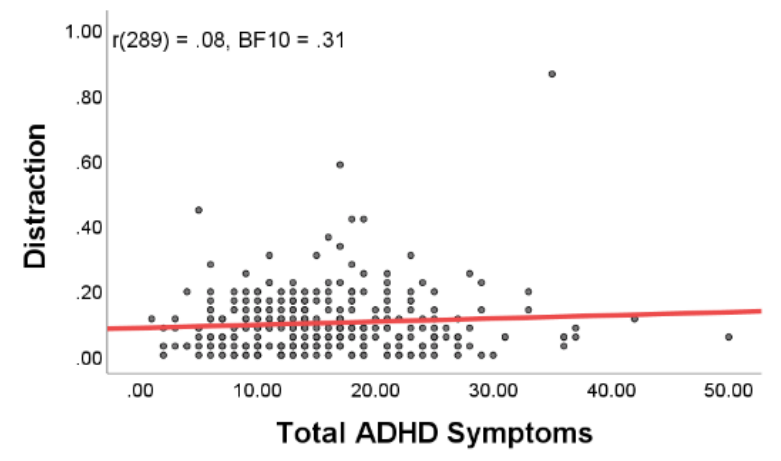

(E)

Figure 1. Correlations of total ADHD symptoms with (a) mind blanking proportion, (b) mind wandering proportion, (c) on-task proportion, (d) task-related interference proportion, and (e) distraction proportion. The red line is the line of best fit. 


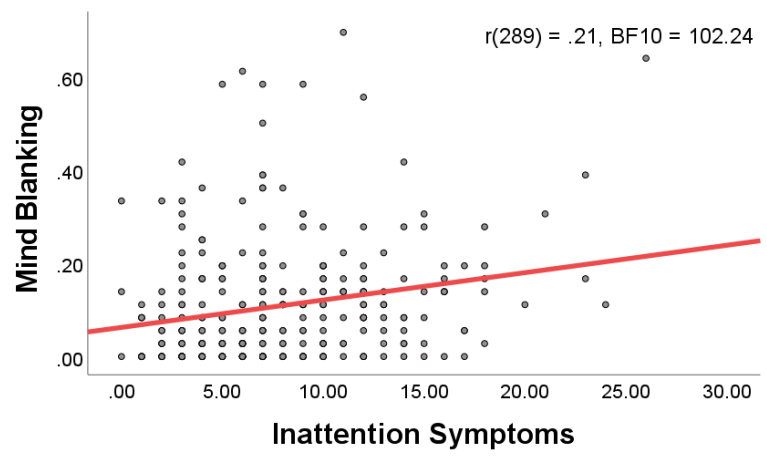

(A)

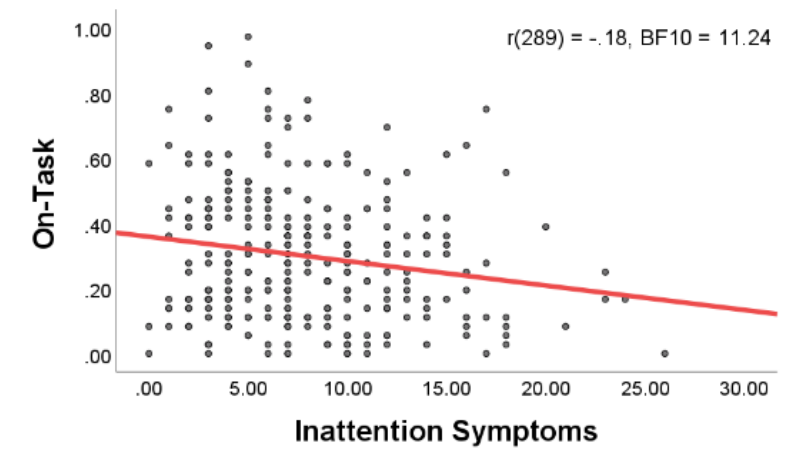

(C)

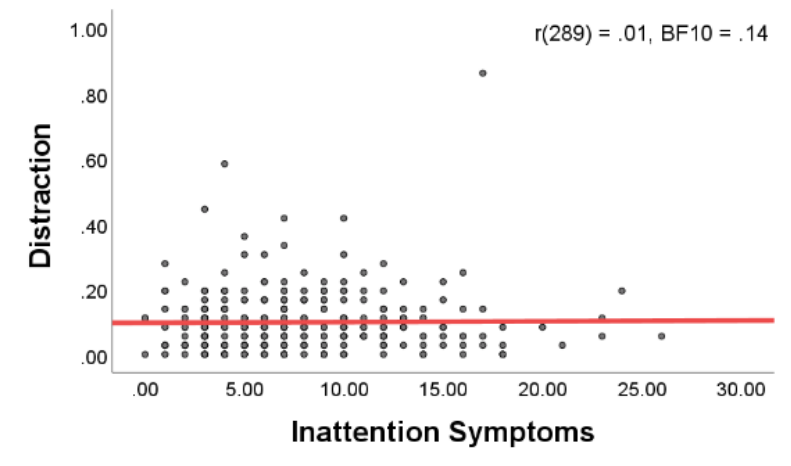

(E)

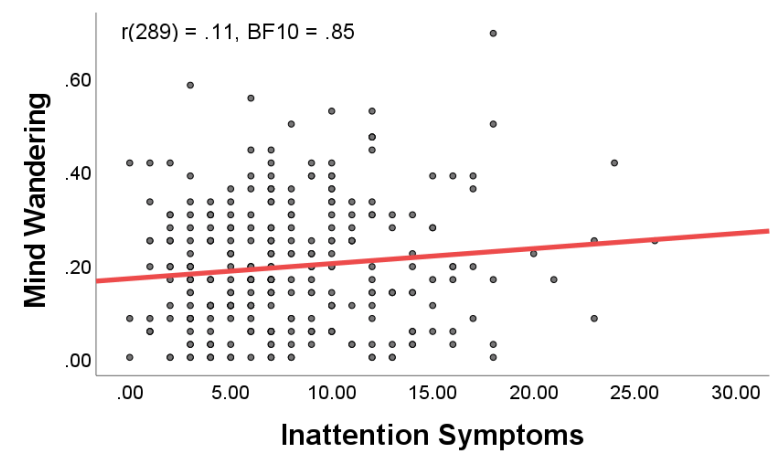

(B)

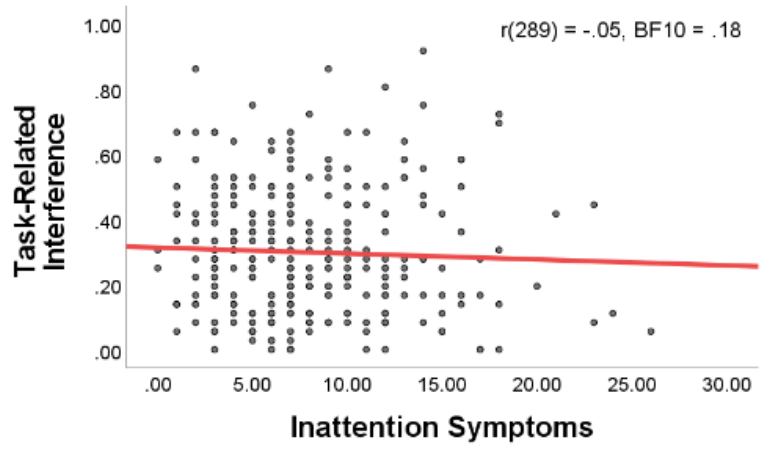

(D) 


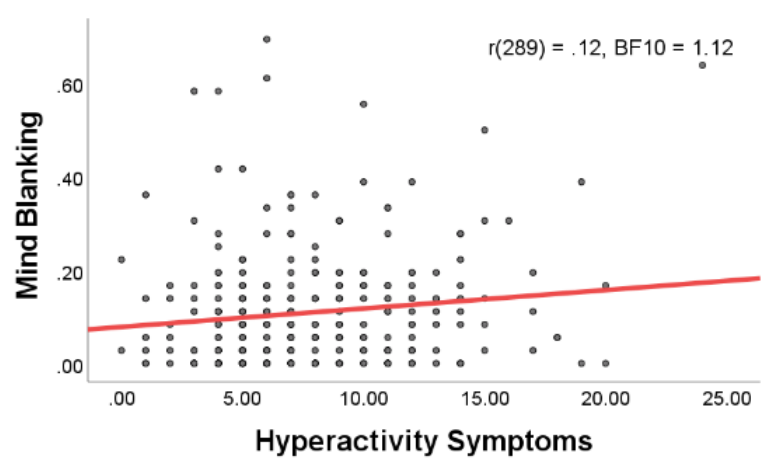

(A)

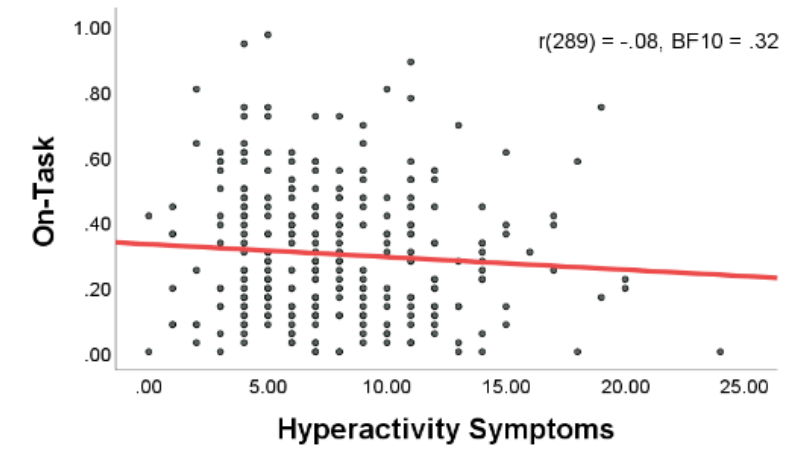

(C)

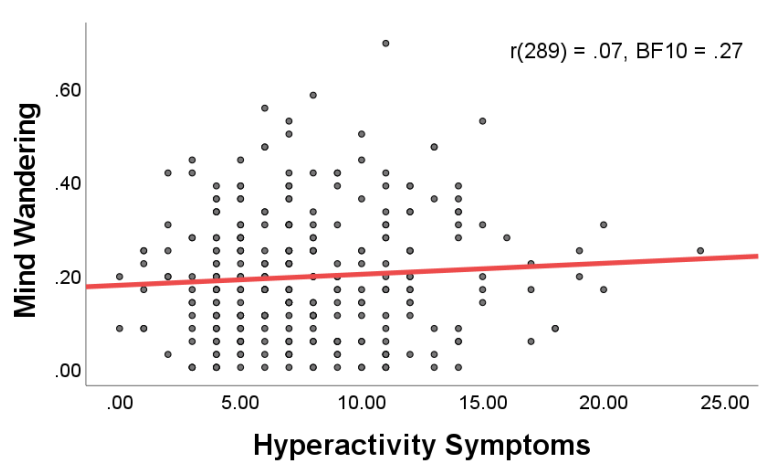

(B)

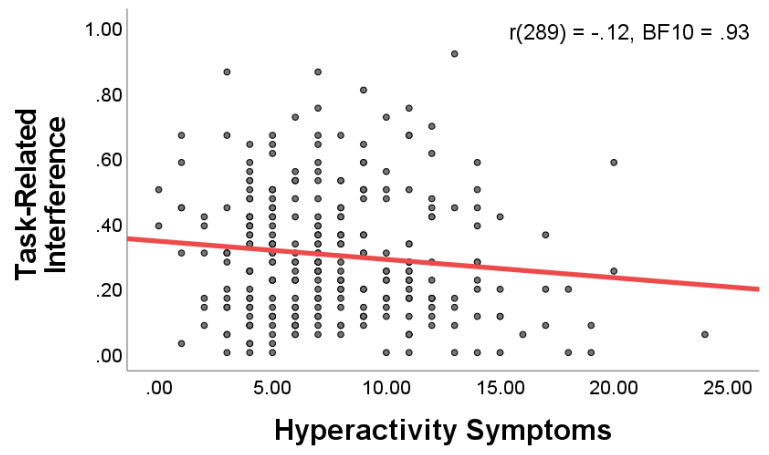

(D)

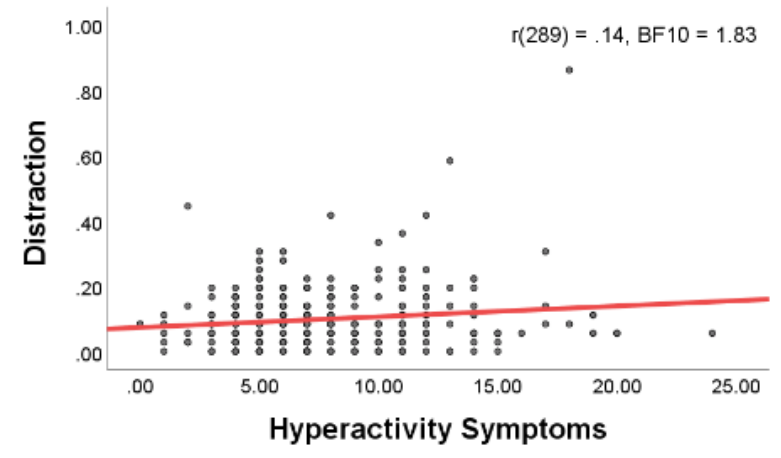

(E)

Figure 3. Correlations of hyperactivity symptoms with (a) mind blanking proportion, (b) mind wandering proportion, (c) on-task proportion, (d) task-related interference proportion, and (e) distraction proportion. The red line is the line of best fit. 


\section{Working Memory Capacity Correlations}

Van den Driessche et al. claimed that mind wandering increased in children with ADHD who were taking medication because they were enabled by the medication to recruit executive resources. This recruitment of executive resources was claimed responsible for greater reports of mind wandering and on-task and fewer reports of mind blanking. However, they did not test executive functioning directly. I correlated mind wandering and mind blanking with working memory capacity (considered a measure of executive ability; Engle, 2002; Burgoyne \& Engle, 2020). All correlations are displayed in scatterplots below (see Figure 4). Mind wandering was not significantly correlated with working memory capacity, $r(288)=.02, p=.79, C I[-.10, .13]$, $B F_{10}=.14$, consistent with the notion that executive resources do not support mind wandering. Mind blanking, $r(288)=-.04, p=.53, C I[-.15, .08], B F_{10}=.17$, along with on-task, $r(288)=.03$, $p=.65, C I[-.09, .14], B F_{10}=.15$, and task-related, $r(288)=.05, p=.38, C I[-.06, .17], B F_{10}=$ .20 , thought reports were also not associated with working memory capacity. Distraction thought reports were negatively associated with working memory capacity, $r(288)=-.13, p=.03, C I[-$ $.24,-.01], B F_{10}=1.32$. As can be seen in Figure 4 Panel E, an outlier may have exerted influence on this estimate. When this outlier was removed, distraction thought reports were no longer significantly associated with working memory capacity, $r(287)=-.09, p=.13, C I[-.20, .03]$, $B F_{10}=.42$. Neither the executive dependent composite, $r(288)=.04, p=.50, C I[-.08, .15], B F_{10}$ $=.17$, nor the non-executive dependent composite were significantly correlated with working memory capacity, $r(288)=-.01, p=.84, C I[-.13, .10], B F_{10}=.14$. 


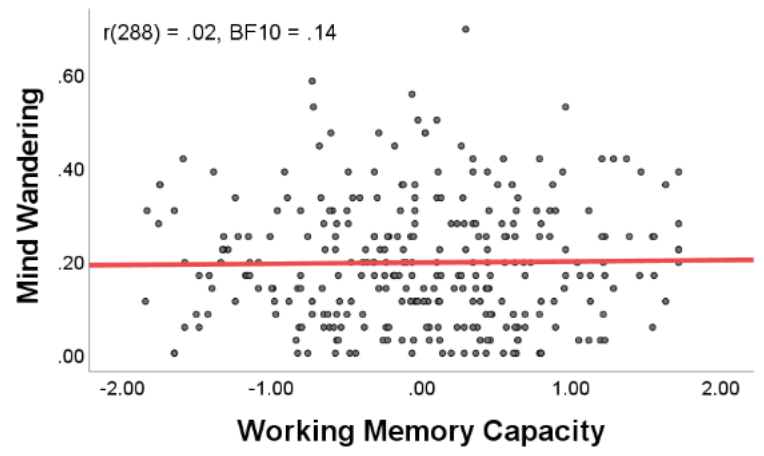

(A)

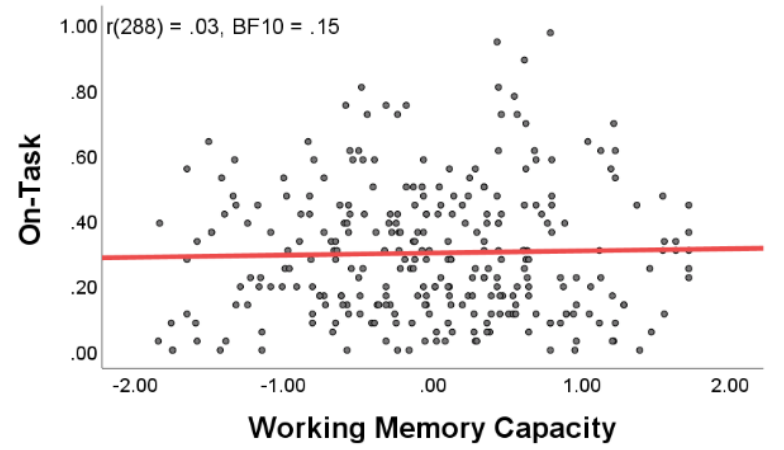

(C)

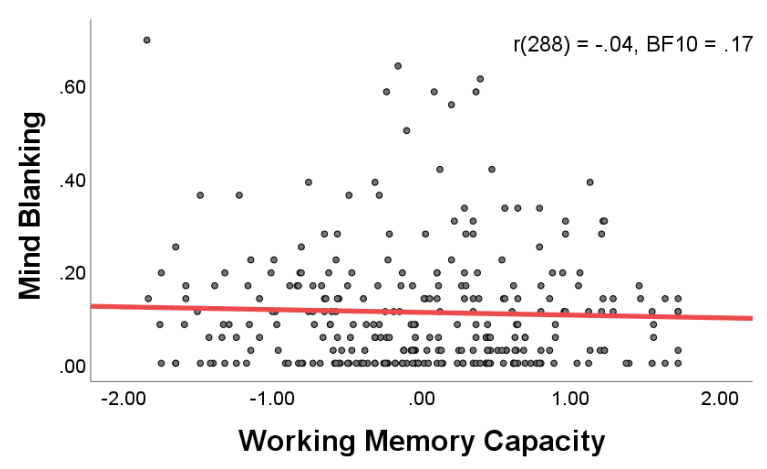

(B)

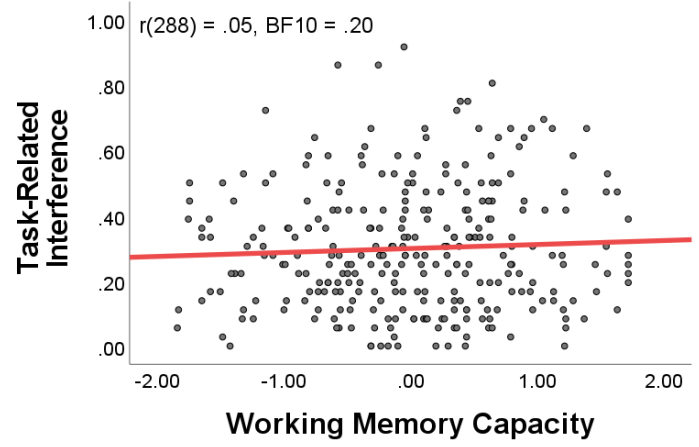

(D)

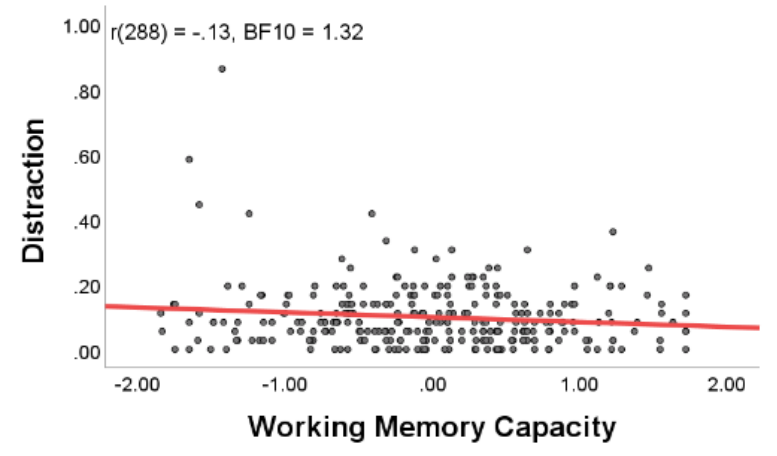

(E)

Figure 4. Correlations of working memory capacity with (a) mind wandering proportion, (b) mind blanking proportion, (c) on-task proportion, (d) task-related interference proportion, and (e) distraction proportion. The red line is the line of best fit. 

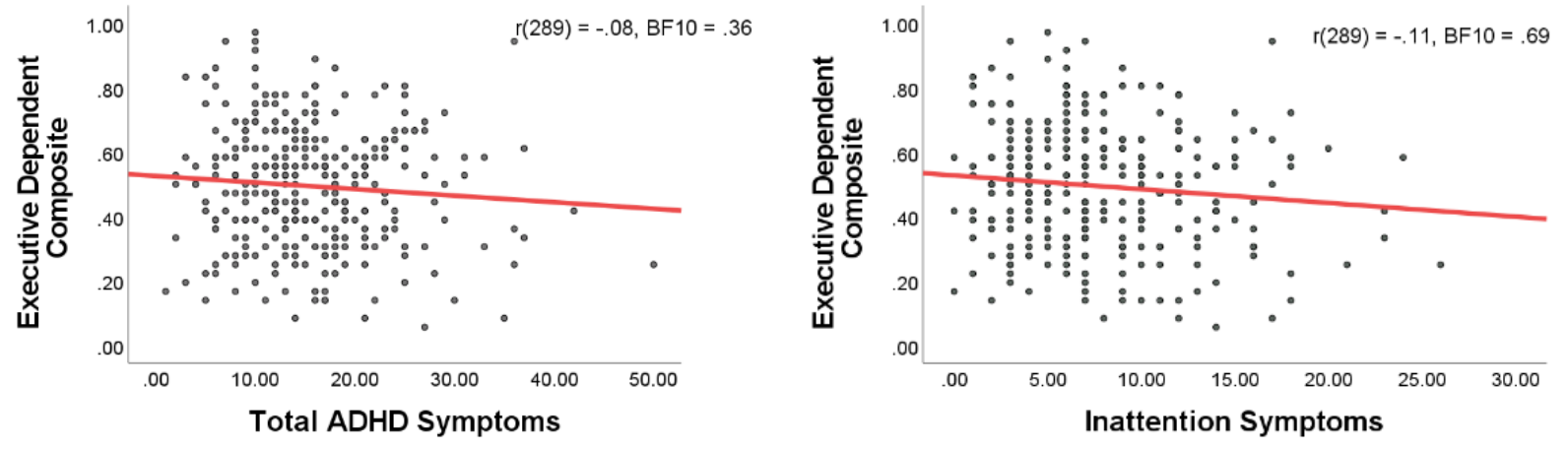

(A)

(B)

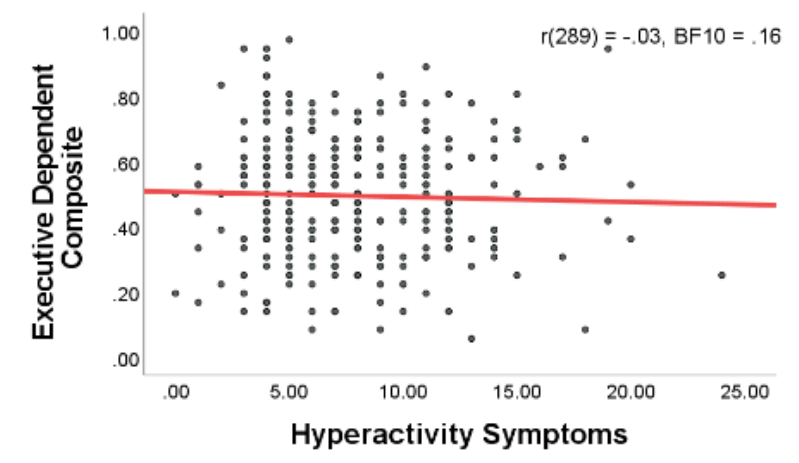

(C)

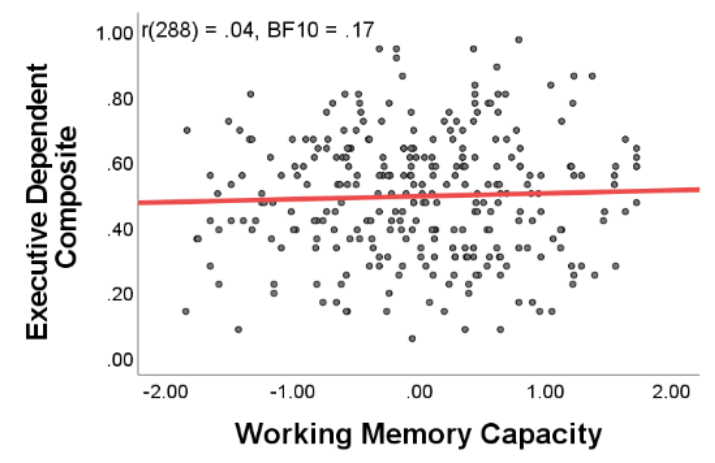

(D)

Figure 5. Correlations of the executive dependent composite (mind wandering, and on-task thought proportions) with the (a) total ADHD symptoms, (c) inattention symptoms, (c) hyperactivity symptoms, and (d) working memory capacity. The red line is the line of best fit. 


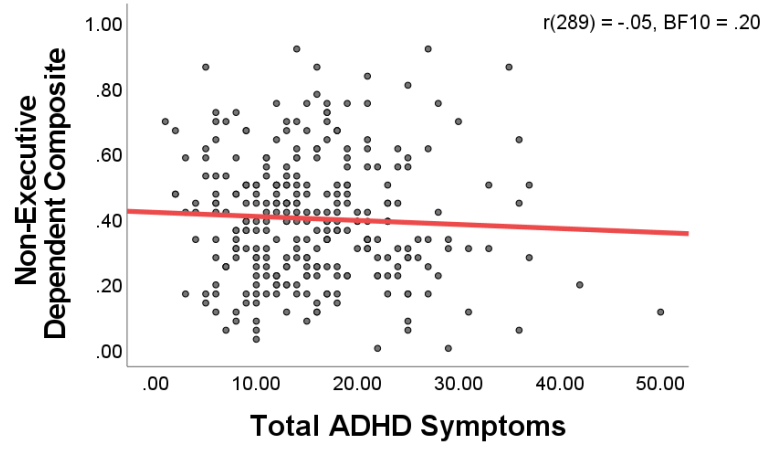

(A)

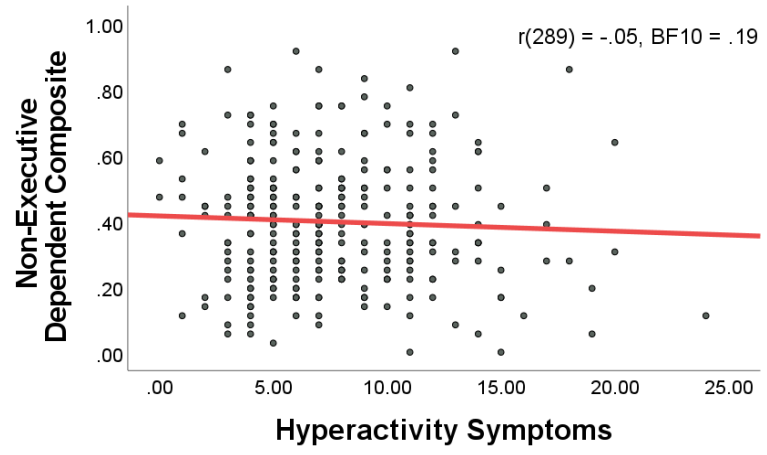

(C)

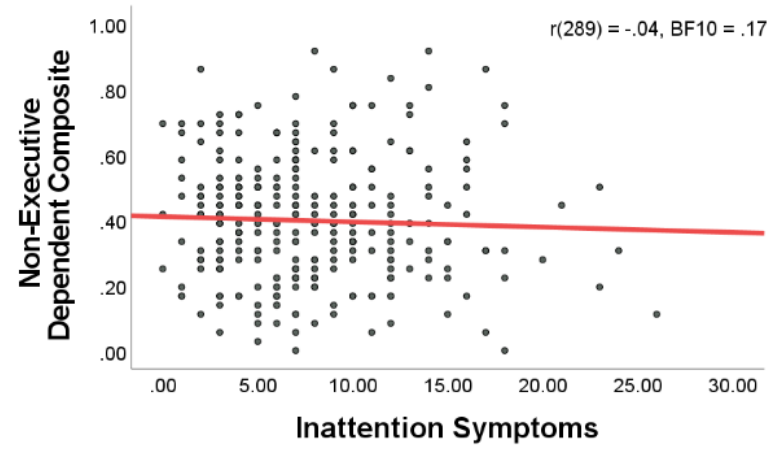

(B)

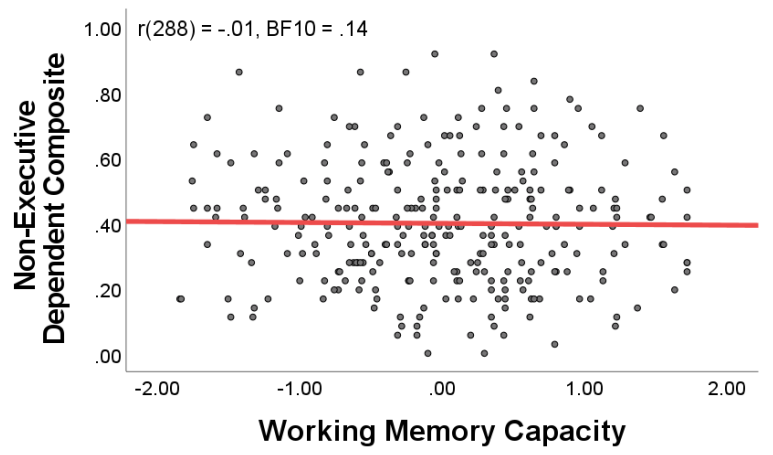

(D)

Figure 6. Correlations of the non-executive dependent (task-related interference and distraction thought proportions) composite with the (a) total ADHD symptoms, (b) inattention symptoms, (c) hyperactivity symptoms, and (d) working memory capacity. The red line is the line of best fit. 


\section{Linear Regression}

To test an alternate model of the relations among ADHD, working memory capacity (executive function) and thought reports to that of the one suggested by Van den Driessche et al, I regressed each thought probe response proportion onto working memory capacity and total ADHD symptoms (see Table 3 for all parameter estimates). More specifically, I examined if working memory capacity moderates the association between ADHD symptoms and thought reports. Consistent with the zero-order correlations, the parameter estimate for total ADHD symptoms was significantly positively associated with mind blanking, $B=-0.33, t(289)=3.39, p$ $<.001, C I[0.001,0.005]$, and negatively associated with on-task thought, $B=-0.39, t(289)=-$ $2.53, p=.01, C I[-0.007,-0.000]$. The interaction of working memory capacity and total ADHD symptoms was not statistically significant in any model: mind blanking, $B=0.13, t(289)=1.08$, $p=.28, C I[-0.001,0.004]$, on-task thought, $B=-0.13, t(289)=-0.66, p=.51, C I[-0.005,0.003]$, distraction, $B=-0.12, t(289)=-1.30, p=.19, C I[-0.003,0.001]$, task interference, $B=0.15$, $t(289)=0.82, p=.41, C I[-0.002,0.005]$, mind wandering, $B=-0.04, t(289)=-0.34, p=.74, C I$ [-0.004 0.002]. In summary, these results provided no evidence for the utility of this alternate model. 
Table 3

Linear Regression of WMC and Total ADHD Symptoms on each Thought Probe Response

\begin{tabular}{|c|c|c|c|c|}
\hline Dependent Variable & Predictor Variables & $B$ & $t$ value & $p$ \\
\hline \multirow[t]{3}{*}{ On-Task } & WMC & 2.69 & 0.80 & .43 \\
\hline & Total ADHD & -0.39 & -2.53 & .01 \\
\hline & WMC x Total ADHD & -0.13 & -0.66 & .51 \\
\hline \multirow[t]{3}{*}{ Task- Related Interference } & WMC & -1.08 & -0.34 & .74 \\
\hline & Total ADHD & -0.22 & -1.48 & .14 \\
\hline & WMC x Total ADHD & 0.15 & 0.82 & .41 \\
\hline \multirow[t]{3}{*}{ Distraction } & WMC & 0.41 & 0.25 & .80 \\
\hline & Total ADHD & 0.10 & 1.30 & .19 \\
\hline & WMC x Total ADHD & -0.12 & -1.30 & .19 \\
\hline \multirow[t]{3}{*}{ Mind Wandering } & WMC & 0.86 & 0.38 & .70 \\
\hline & Total ADHD & 0.18 & 1.75 & .08 \\
\hline & WMC x Total ADHD & -0.04 & -0.34 & .74 \\
\hline \multirow[t]{3}{*}{ Mind Blanking } & WMC & -2.72 & -1.27 & .21 \\
\hline & Total ADHD & 0.33 & 3.39 & .00 \\
\hline & WMC x Total ADHD & 0.13 & 1.08 & .28 \\
\hline \multirow[t]{3}{*}{ Exec Dependent Composite } & WMC & 3.55 & 1.12 & .27 \\
\hline & Total ADHD & -0.21 & -1.44 & .15 \\
\hline & WMC x Total ADHD & -0.17 & -0.94 & .35 \\
\hline \multirow[t]{2}{*}{ Non-Exec Dependent Composite } & WMC & -0.68 & -0.21 & .83 \\
\hline & Total ADHD & -0.12 & -0.83 & .40 \\
\hline
\end{tabular}




$$
\text { WMC x Total ADHD } \quad 0.03 \quad 0.17 \quad .86
$$

Note: $\mathrm{WMC}=$ Working Memory Capacity; ADHD = Attention Deficit/Hyperactivity Disorder; Exec $=$ Executive. 


\section{CHAPTER FOUR: DISCUSSION}

Van den Driessche et al. (2017) examined subjective thought reports in their subjects, including a subset of children diagnosed with ADHD and young adults with elevated ADHD symptoms. Van den Driessche et al. reported three key findings (they determined what was key, not I). First, mind blanking was elevated in populations with clinical and subclinical ADHD. Second, in both of Van den Driessche et al.'s experiments, the increase of mind blanking happened at the expense of fewer reports of mind wandering and on-task thoughts (as measured by the executive dependent composite). Lastly, for the medicated subjects in Experiment 1, methylphenidate increased mind wandering, which Van den Driessche suspected was due to recruited executive resources. However, Van den Driessche et al. provided no direct empirical evidence for this claim. They inferred a causal relation between executive function and thought reports primarily from the increased mind wandering in the medicated subjects.

The current study corroborated Van den Driessche et al.'s first key finding; there was a statistically significant positive correlation between mind blanking and total ADHD symptomology. The combination of a positive ADHD-mind blanking association and a negative association between ADHD and on-task reports also is broadly consistent with the trade-off reported by Van den Driessche et al.'s second key finding. But here, importantly, this trade-off differs from Van den Driessche et al. because it does not involve mind wandering and mind blanking. I did not corroborate the third key inference from Van den Driessche et al. They speculated that because of a lack of executive resources, mind blanking increased at the expense

of mind wandering and on-task thoughts and that medication recruited executive resources, thus decreasing mind blanking and increasing mind wandering and on-task reports. Here, ADHD 
symptomology was not associated with the executive dependent composite or mind wandering and no evidence was detected of associations between working memory capacity (my executive resources measure) and mind blanking, mind wandering or the (presumed) executive dependent composite.

Given that this pattern of results is inconsistent with the broad claim of executive functioning being responsible for ADHD-related mind blanking and supporting mind wandering and on-task thought, alternate explanations are suggested. One possibility is that people with more ADHD symptoms may either have metacognitive deficits or accurately report no thought content. As mentioned in the introduction, metacognitive deficits in those with ADHD symptoms have been reported (Antshel \& Natasi, 2008; Franklin et al., 2017; Knouse et al., 2005). Motivation is another plausible factor that may play a role in determining though reports as mentioned (but not seriously considered) by Van den Driessche et al. Motivation is lower in those with ADHD (Volkow et al., 2011) and may be required to sustain attention (on a task).

When results from two studies are inconsistent with each other, methodological differences should be noted and considered. First, the sample I tested did not have formal ADHD diagnoses, unlike the ADHD groups from Van den Driessche et al.'s Experiment 1. However, the ADHD self-report measure used in the current study, the ADHDRS-IV, was created using DSMIV diagnostic criteria for ADHD (Collett et al., 2003) and should roughly align with ADHD diagnosis. Furthermore, ADHD is dimensional (Haslam et al., 2020) indicating that treating ADHD symptomology as a continuous measure like I did (unlike Van den Driessche et al. who in both of their experiments dichotomized ADHD) is justified. Another difference is that I only used young adult subjects, whereas Van den Driessche et al. used children in Experiment 1 (and young adults in Experiment 2). Their Experiment 1 findings were the basis for the overarching 
claim that mind wandering requires executive resources. Previous research has provided evidence that ADHD in childhood and adulthood are distinct disorders (Agnew-Blais et al., 2016; Caye et al., 2016). Van den Driessche et al.'s claim that executive resources support mind wandering is perhaps too broad (i.e., they overgeneralized). It may be more accurate to make more specific claims to the age range of their experiments' samples. For example, they may be on more firm footing with this inference if they put (at least) an age constraint on its generalizability (Simons, Shoda, \& Lindsay, 2017; Yarkoni, 2020).

The current study has clinical implications, specifically with regards to strengthening the therapeutic alliance. There is a positive association between the therapeutic alliance quality and various therapy outcomes (Horvath et al., 2011; Horvath \& Luborksy, 1993). This study corroborates findings from Van den Driessche et al. about the conscious experience of people with ADHD symptomology. Gaining insight into someone's conscious experience can help practitioners better understand and identify with their clients. This knowledge can enhance rapport and supportive techniques such as empathetic, validating, and understanding comments that are essential for creating and maintaining a solid therapeutic alliance (Leibovich et al., 2020).

\section{Conclusion}

The current study corroborated two of Van den Driessche et al.'s key findings while providing evidence inconsistent with another. Corroborating Van den Driessche et al., a statistically significant positive association between mind blanking propensity and total ADHD symptomology suggests that those with more ADHD symptomology have experiences of a blank mind more often than those with less ADHD symptomology. Along with this positive ADHDmind blanking association, higher ADHD symptomology was associated with fewer on-task 
reports consistent with Van den Driessche et al.'s reporting of a trade-off. However, the trade-off differs here because it did not involve mind wandering. I found no evidence for an association between total ADHD symptomology and the executive-dependent composite or mind wandering. There was also no evidence for an association between working memory capacity, mind blanking, mind wandering, and the executive-dependent composite. These latter two results, the lack of a trade-off involving mind wandering, and the null correlations among ADHD, mind wandering, and the executive composite provide no evidence for executive resources supporting mind wandering. These latter two results also conflict with the claim of the role of executive functions in the conscious experience of those with ADHD made by Van den Driessche et al. 


\section{REFERENCES}

American Psychiatric Association. (2013). Neurodevelopmental disorders. In Diagnostic and statistical manual of mental disorders (5th ed.).

Agnew-Blais, J. C., Polanczyk, G. V., Danese, A., Wertz, J., Moffitt, T. E., \& Arseneault, L. (2016). Evaluation of the persistence, remission, and emergence of attentiondeficit/hyperactivity disorder in young adulthood. JAMA Psychiatry, 73(7), 713.

Antshel, K. M., \& Nastasi, R. (2008). Metamemory development in preschool children with ADHD. Journal of Applied Developmental Psychology, 29(5), 403-411.

Barkley, R. A. (1997). Attention-deficit/hyperactivity disorder, self-regulation, and time: Toward a more comprehensive theory. Journal of Developmental and Behavioral Pediatrics: JDBP, $18(4), 271-279$.

Caye, A., Rocha, T. B.-M., Anselmi, L., Murray, J., Menezes, A. M. B., Barros, F. C., Gonçalves, H., Wehrmeister, F., Jensen, C. M., Steinhausen, H.-C., Swanson, J. M., Kieling, C., \& Rohde, L. A. (2016). Attention-deficit/hyperactivity disorder trajectories from childhood to young adulthood: Evidence from a birth cohort supporting a late-onset syndrome. JAMA Psychiatry, 73(7), 705.

Collett, B. R., Ohan, J. L., \& Myers, K. M. (2003). Ten-year review of rating scales. V: Scales assessing attention-deficit/hyperactivity disorder. Journal of the American Academy of Child \& Adolescent Psychiatry, 42(9), 1015-1037.

Conway, A. R. A., Kane, M. J., Bunting, M. F., Hambrick, D. Z., Wilhelm, O., \& Engle, R. W. (2005). Working memory span tasks: A methodological review and user's guide. Psychonomic Bulletin \& Review, 12(5), 769-786. 
DuPaul, G. J., Power, T. J., Anastopoulos, A. D., \& Reid, R. (2016). ADHD rating scale - 5 for children and adolescents: Checklists, norms, and clinical interpretation. The Guilford Press.

Foster, J. L., Shipstead, Z., Harrison, T. L., Hicks, K. L., Redick, T. S., \& Engle, R. W. (2015). Shortened complex span tasks can reliably measure working memory capacity. Memory \& Cognition, 43(2), 226-236.

Franklin, M. S., Mrazek, M. D., Anderson, C. L., Johnston, C., Smallwood, J., Kingstone, A., \& Schooler, J. W. (2017). Tracking distraction: The relationship between mind wandering, meta-awareness, and ADHD symptomatology. Journal of Attention Disorders, 21(6), 475486.

Haslam, N., McGrath, M. J., Viechtbauer, W., \& Kuppens, P. (2020). Dimensions over categories: A meta-analysis of taxometric research. Psychological Medicine, 50(9), 14181432.

Haenlein, M., \& Caul, W. F. (1987). Attention deficit disorder with hyperactivity: A specific hypothesis of reward dysfunction. Journal of the American Academy of Child \& Adolescent Psychiatry, 26(3), 356-362.

Horvath, A. O., Del Re, A. C., Flückiger, C., \& Symonds, D. (2011). Alliance in individual psychotherapy. Psychotherapy, 48(1), 9-16.

Horvath, A. O., \& Luborsky, L. (1993). The role of the therapeutic alliance in psychotherapy. Journal of Consulting and Clinical Psychology, 61(4), 561-573.

JASP Team (2020). JASP (Version 0.14.1)[Computer software]. 
Johansen, E., Killeen, P. R., Russell, V. A., Tripp, G., Wickens, J. R., Tannock, R., Williams, J., \& Sagvolden, T. (2009). Origins of altered reinforcement effects in ADHD. Behavioral and Brain Functions, 5(1), 7.

Kane, M. J., Hambrick, D. Z., Tuholski, S. W., Wilhelm, O., Payne, T. W., \& Engle, R. W. (2004). The generality of working memory capacity: A latent-variable approach to verbal and visuospatial memory span and reasoning. Journal of Experimental Psychology: General, 133(2), 189-217.

Kane, M. J., Brown, L. H., McVay, J. C., Silvia, P. J., Myin-Germeys, I., \& Kwapil, T. R. (2007). For whom the mind wanders, and when: an experience-sampling study of working memory and executive control in daily life. Psychological Science, 18(7), 614-621.

Kane, M. J., Meier, M. E., Smeekens, B. A., Gross, G. M., Chun, C. A., Silvia, P. J., \& Kwapil, T. R. (2016). Individual differences in the executive control of attention, memory, and thought, and their associations with schizotypy. Journal of Experimental Psychology: General, 145(8), 1017-1048.

Kane, M. J., Gross, G. M., Chun, C. A., Smeekens, B. A., Meier, M. E., Silvia, P. J., \& Kwapil, T. R. (2017). For whom the mind wanders, and when, varies across laboratory and daily-life settings. Psychological Science, 28(9), 1271-1289.

Knouse, L. E., Bagwell, C. L., Barkley, R. A., \& Murphy, K. R. (2005). Accuracy of selfevaluation in adults with ADHD: Evidence from a driving study. Journal of Attention Disorders, 8(4), 221-234.

Kooij, J. J. S., \& Francken, M. H. (2010). DIVA 2.0: Diagnostic Interview for ADHD in adults. Retrieved from http://www.divacenter.eu/Content/VertalingPDFs/diva_2_nl_formulier.pdf 
Leibovich, L., Front, O., McCarthy, K. S., \& Zilcha-Mano, S. (2020). How do supportive techniques bring about therapeutic change: The role of therapeutic alliance as a potential mediator. Psychotherapy, 57(2), 151-159.

Levinson, D. B., Smallwood, J., \& Davidson, R. J. (2012). The persistence of thought: Evidence for a role of working memory in the maintenance of task-unrelated thinking. Psychological Science, 23(4), 375-380.

McCabe, D. P., Roediger, H. L., McDaniel, M. A., Balota, D. A., \& Hambrick, D. Z. (2010). The relationship between working memory capacity and executive functioning: Evidence for a common executive attention construct. Neuropsychology, 24(2), 222-243.

McVay, J. C., \& Kane, M. J. (2009). Conducting the train of thought: Working memory capacity, goal neglect, and mind wandering in an executive-control task. Journal of Experimental Psychology: Learning, Memory, and Cognition, 35(1), 196-204.

McVay, J. C., \& Kane, M. J. (2010). Does mind wandering reflect executive function or executive failure? Comment on Smallwood and Schooler (2006) and Watkins (2008). Psychological Bulletin, 136(2), 188-197.

McVay, J. C., \& Kane, M. J. (2012). Why does working memory capacity predict variation in reading comprehension? On the influence of mind wandering and executive attention. Journal of Experimental Psychology: General, 141(2), 302-320.

McVay, J. C., \& Kane, M. J. (2012b). Drifting from slow to “d'oh!”: Working memory capacity and mind wandering predict extreme reaction times and executive control errors. Journal of Experimental Psychology: Learning, Memory, and Cognition, 38(3), 525-549. 
Meier, M. E. (2019). Is there a positive association between working memory capacity and mind wandering in a low-demand breathing task? A preregistered replication of a study by Levinson, Smallwood, and Davidson (2012). Psychological Science, 30(5), 789-797.

Mrazek, M. D., Smallwood, J., Franklin, M. S., Chin, J. M., Baird, B., \& Schooler, J. W. (2012). The role of mind-wandering in measurements of general aptitude. Journal of Experimental Psychology: General, 141(4), 788-798.

Psychology Software Tools, Inc. [E-Prime 2.0]. (2012). Retrieved from http://www.pstnet.com.

Robison, M. K., \& Unsworth, N. (2018). Cognitive and contextual correlates of spontaneous and deliberate mind wandering. Journal of Experimental Psychology: Learning, Memory, and Cognition, 44(1), 85-98.

Simons, D. J., Shoda, Y., \& Lindsay, D. S. (2017). Constraints on Generality (COG): A proposed addition to all empirical papers. Perspectives on Psychological Science, 12(6),11231128.

Smallwood, J. (2010). Why the global availability of mind wandering necessitates resource competition: Reply to McVay and Kane (2010). Psychological Bulletin, 136(2), 202-207.

Smallwood, J. (2013). Distinguishing how from why the mind wanders: A process-occurrence framework for self-generated mental activity. Psychological Bulletin, 139(3), 519-535.

Smallwood, J. \& Schooler, J. (2015). The science of mind wandering: Empirically navigating the stream of consciousness. Annual Review of Psychology, 66, 487-518.

Unsworth, N., Heitz, R. P., Schrock, J. C., \& Engle, R. W. (2005). An automated version of the operation span task. Behavior Research Methods, 37(3), 498-505.

Unsworth, N., \& McMillan, B. D. (2013). Mind wandering and reading comprehension: Examining the roles of working memory capacity, interest, motivation, and topic 
experience. Journal of Experimental Psychology: Learning, Memory, and Cognition, $39(3), 832-842$.

Unsworth, N., \& McMillan, B. D. (2014). Similarities and differences between mind wandering and external distraction: A latent variable analysis of lapses of attention and their relation to cognitive abilities. Acta Psychologica, 150, 14-25.

Unsworth, N., \& Robison, M. K. (2020). Working memory capacity and sustained attention: A cognitive-energetic perspective. Journal of Experimental Psychology: Learning, Memory, and Cognition, 46(1), 77-103.

Van den Driessche, C., Bastian, M., Peyre, H., Stordeur, C., Acquaviva, É., Bahadori, S., Delorme, R., \& Sackur, J. (2017). Attentional lapses in attention-deficit/hyperactivity disorder: Blank rather than wandering thoughts. Psychological Science, 28(10), 1375-1386.

Volkow, N. D., Wang, G.-J., Newcorn, J. H., Kollins, S. H., Wigal, T. L., Telang, F., Fowler, J. S., Goldstein, R. Z., Klein, N., Logan, J., Wong, C., \& Swanson, J. M. (2011). Motivation deficit in ADHD is associated with dysfunction of the dopamine reward pathway. Molecular Psychiatry, 16(11), 1147-1154.

Ward, A. F., \& Wegner, D. M. (2013). Mind blanking: When the mind goes away. Frontiers in Psychology, 4.

Yarkoni, T. (2020). The generalizability crisis. Behavioral and Brain Sciences, 1-37. 


\section{Appendix A}

Operation Span Complex Span Task (Unsworth et al., 2005).

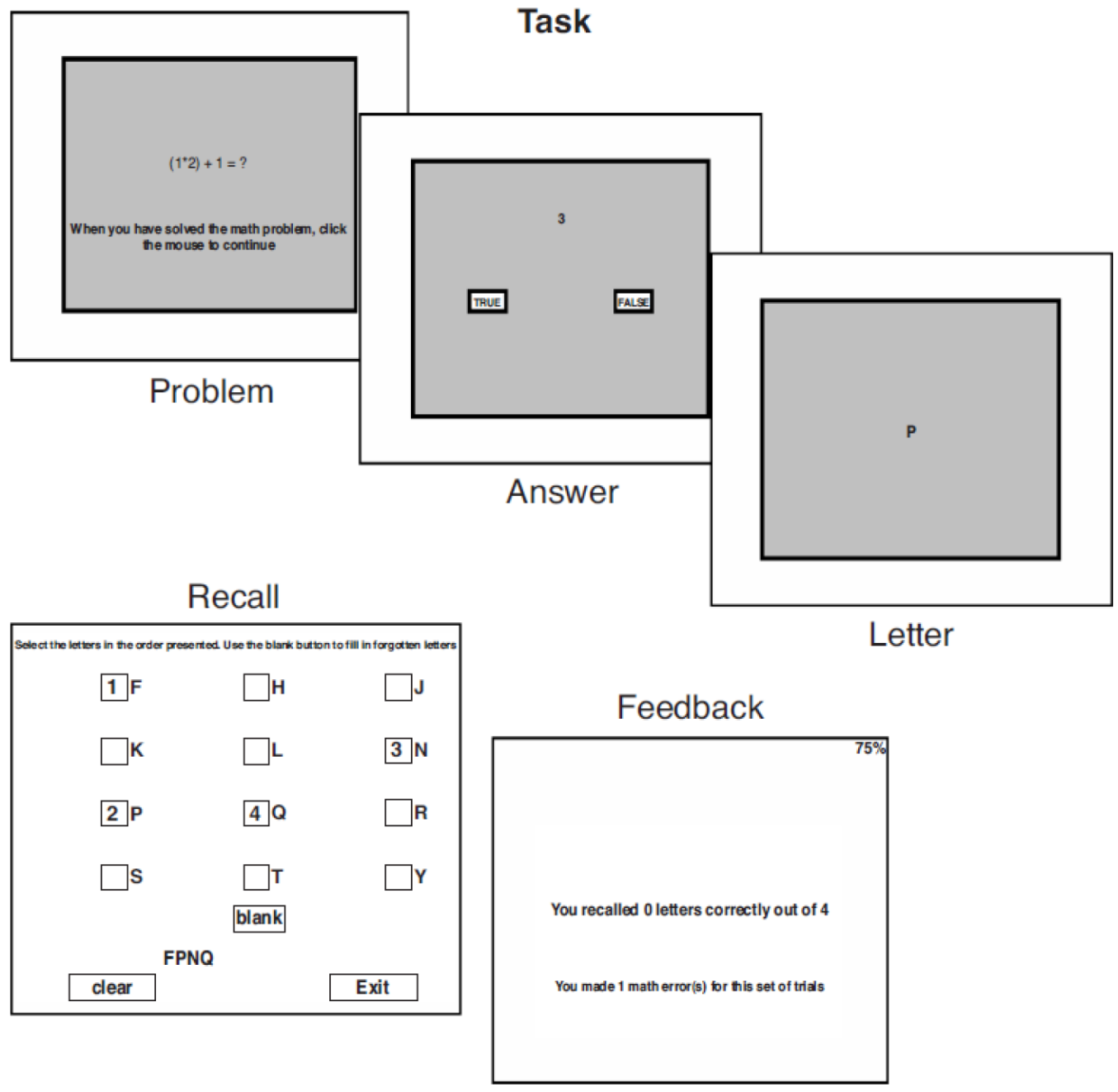

Figure A. A diagram depicting how subjects completed the operation span task. 


\section{Appendix B}

Symmetry Span Complex Span Task (Kane et al., 2004)

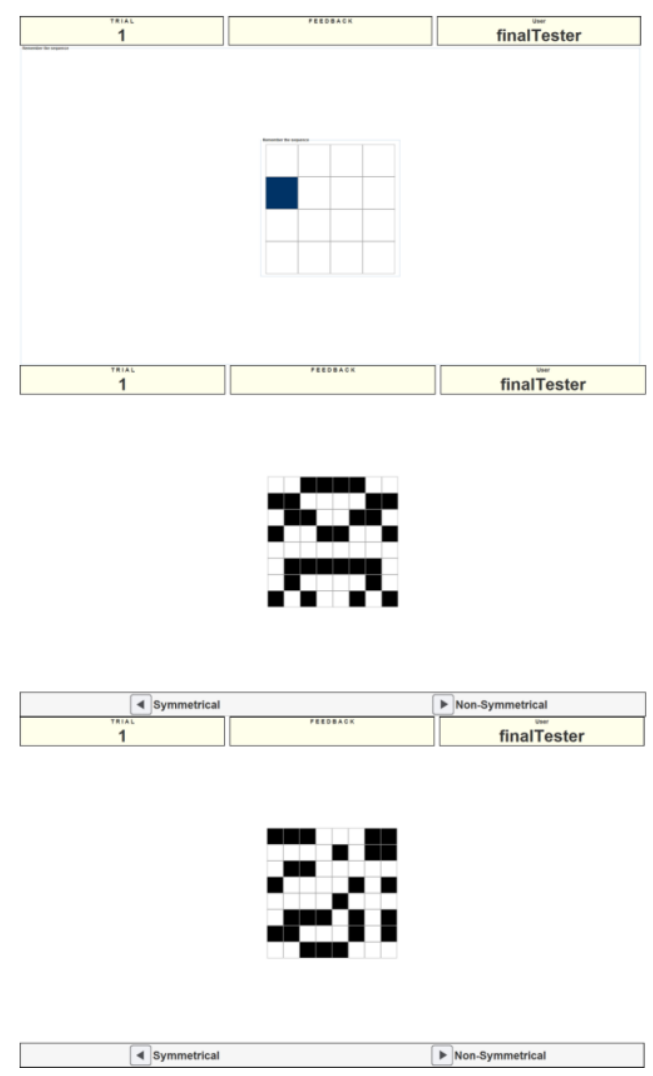

Figure B1. The presentation phase of the symmetry span task.

\begin{tabular}{|c|c|}
\hline $1^{\text {TRIAL }}$ & fHosck \\
\hline
\end{tabular}

Figure B2. The recall phase of the symmetry span task. 


\section{Appendix C}

Sustained Attention to Response Task (SART; Kane et al., 2016)

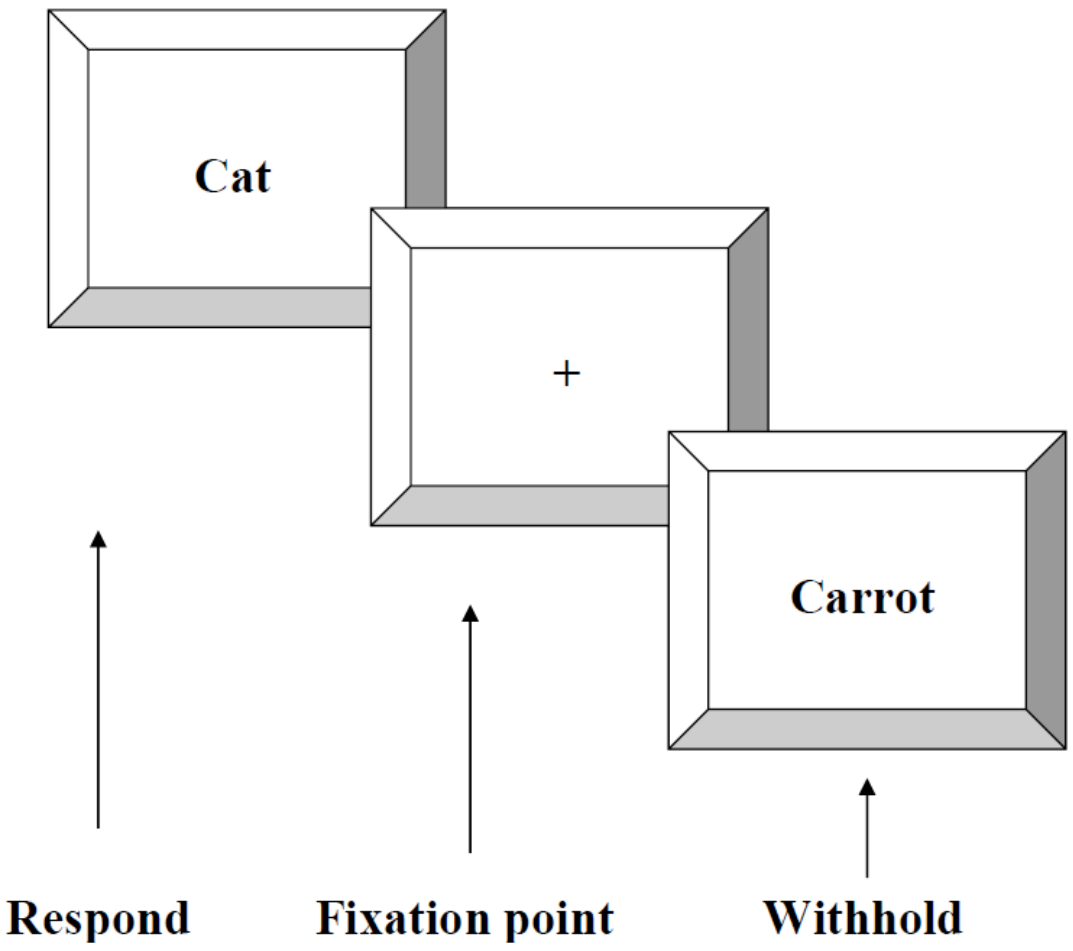

Figure $C$. A diagram depicting the response and withhold section of the SART. 


\section{Appendix D}

\section{Sustained Attention to Response Task (SART) Instructions}

As you are performing the task, you will periodically see a screen like this:

It will ask:

What were you just thinking about?

Please press a number on the keyboard.

1. The task

2. Task experience or performance

3. Distraction

4. Mind Wandering

5. Mind Blanking

Press the space bar to continue...

Screen 2: When you are asked what you are thinking, please take a moment to reflect on your thoughts before you answer.

The choices are:

1. The task

Select this number if your thoughts were about the words or categories of words that you just saw.

Press the space bar to continue.

Screen 3: 2. Task experience or performance 
Select this number if your thoughts were about how well or poorly you are doing on the task or how many you are getting right or wrong.

Press the space bar to continue...

Screen 4: 3. Distraction

Select this number if your thoughts were about a noise, smell (or something else) in your environment that makes you think about what is happening around you.

Press the space bar to continue...

\section{Screen 5: 4. Mind Wandering}

Select this number if your thoughts were not about the task or what's happening around you, but your thoughts are about something else. For example thinking about something that has happened to you in the past, something you're going to do in the future, or any other thoughts that are not about the task and not about some distraction in your environment.

Press the space bar to continue...

Screen 6:

\section{Mind Blanking}

Select this number if you are not thinking about the task or anything else. Your mind was blank. Press the space bar to continue...

\section{Screen 7:}


Remember, when you see screens like these, please respond based on what you were thinking *just before* the screen appeared. Do not try to reconstruct what you were thinking during the preceding words on the screen, and please select the categories that best describes your thoughts as accurately as you can. Remember that it is quite normal to have any of these kinds of thoughts during an ongoing task.

If you have any questions, please ask me now.

Press the space bar to continue... 


\section{Appendix E}

ADHDRS-IV (Dupaul et al., 1998) Self-Report Questionnaire

Within the past two weeks rate on a scale from never to very often how much you have experience the following:

1. Carelessness

2. Difficulty sustaining attention in activities

3. Doesn't listen

4. No follow through

5. Can't organize

6. Avoids/dislikes tasks requiring sustained mental effort

7. Loses important items

8. Easily distractible

9. Forgetful in daily activities

10. Squirms and fidgets

11. Can’t stay seated

12. Runs/climbs excessively

13. Can’t play/work quietly

14. On the go, "driven by a motor"

15. Talks excessively

16. Blurts out answers

17. Can't wait for turn

18. Intrudes/interrupts others 


\section{Appendix F}

\section{Demographic Questions}

Screen 1: We ask about Age, Sex, Ethnicity, Race, and Handedness in order to report the overall characteristics and diversity of our sample to other scientists and our funding agencies.

We do NOT analyze our data separately for different groups.

Although we would appreciate your responses to all of these questions, you may elect not to answer any of the questions by just pressing ENTER without entering a value.

Press any key...

Screen 2: Please indicate your SEX by typing:

$0=$ male

$1=$ female

Press ENTER.

Screen 3: Please type in your AGE in years.

Press ENTER.

Screen 4: Please indicate your ETHNICITY by typing:

$1=$ Hispanic or Latino

$0=$ not Hispanic or Latino 


\section{Press ENTER.}

Screen 5: Please indicate your RACE by typing:

$0=$ White: European or Middle Eastern descent

$1=$ Asian

$2=$ Black: African or Caribbean descent

3 = Native American or Alaskan Native

$4=$ Native Hawaiian or Pacific Islander

$5=$ Multiracial

$6=$ Other

Press ENTER.

Screen 6: Please indicate your HANDEDNESS by typing:

$0=$ Right-handed

$1=$ Left-handed

Press ENTER. 